\title{
Aktuelle Themen der Flugmedizin
}

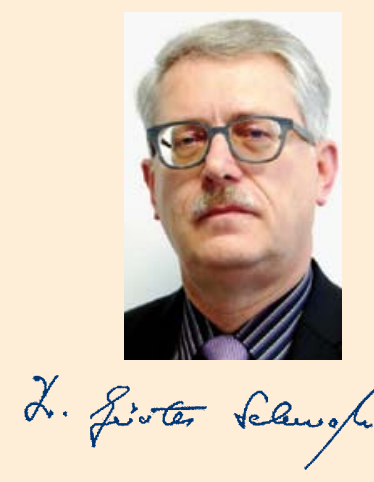

Sehr verehrte Frau Kollegin, sehr geehrter Herr Kollege,

die erste Ausgabe der FTR im neuen Jahr greift wieder aktuelle, zum Teil seit langem diskutierte Themen auf. Die Kasuistik, vorgestellt von Elisabeth Heyn von Aiut Alpin Dolomites, lässt uns an einer schwierigen Bergrettung aus der Großen Zinne im Grödnertal teilhaben. Das Meldebild „Bergsteiger mit Herz-Kreislauf-Problemen“ löst eine Rettungsaktion am Fixtau aus, die nur durch großes Können und viel Erfahrung zu einem guten Ende führt. Zeit bedeutet am Berg Leben. Übrigens: Der Vergleich mit den Bergrettungsaktionen in entsprechenden TV-Serien lohnt sich.

Bereits seit längerer Zeit versuchen große Kohortenstudien Zusammenhänge zwischen der Strahlenexposition des fliegenden Personals und gesundheitlichen Auswirkungen zu klären. Dabei geht es vorrangig um die Frage nach einer erhöhten Krebsinzidenz und Mortalität. Allein in Deutschland gehören einige Zehntausend zum fliegenden Dienst. Die Belastungsfaktoren sind bekannt: Neben der kosmischen Strahlung, deren mögliche gesundheitliche Folgen im Aufsatz von Hajo Zeeb et al., Bremen, betrachtet werden, sind es die Störungen der zirkadianen Rhythmik und die nicht ausgewogene Work-Life-Balance, außerdem der Klimawechsel und die Luftqualität an Bord. Die Ergebnisse der Studien sind differenziert zu betrachten. Bei der Brustkrebsinzidenz des Kabinenpersonals zeigen zum Beispiel die Ergebnisse der deutschen Kohorte keine signifikante Erhöhung, auffällig dagegen das um 50\% erhöhte Risiko beim nordischen Kabinenpersonal. Ob Störungen des Schlaf-Wach-Rhythmus gesundheitliche Langzeitfolgen haben, lässt sich aus den vorliegenden Studien nicht ableiten.

Mit auffälligen Gerüchen in Flugzeugen und damit im Zusammenhang stehenden Gesundheitsbeschwerden der Besatzungen berichten Christian Felten et al., Bochum. Obwohl Zersetzungsprodukte von Turbinenöle und das Additiv Trikresylphosphat (TCP) nachweislich in Kabine und Cockpit eindringen können, ist bei den geklagten Beschwerden des fliegenden Personals von einem multifaktoriellen Geschehen auszugehen; offenbar gibt es zahlreiche Quellen für Störgerüche. Gesundheitsadverse Effekte durch TCP allein können jedenfalls ausgeschlossen werden. In einer Übersichtsarbeit stellen Philipp Stahl und Ralph T. Schwarz, Marburg, die aktuellen Erkenntnisse und Wissensdefizite der Chagaskrankheit vor. 8-10 Mio. Menschen leiden derzeit weltweit an dieser zu wenig im Fokus der Forschung stehenden Zoonose. 100 Mio. Menschen sind potenziell gefährdet, insbesondere in Mittel- und Südamerika, da wo Raubwanzen als Überträger leichten Zugang haben. Eine Impfung existiert nicht. Die beiden auf dem Markt befindlichen Medikamente sind schwer erhältlich und nebenwirkungsreich. Präventiv muss auf die Expositionsprophylaxe durch Anwendung von Insektiziden, geeignete Kleidung und das Erkennen infizierter Haustiere geachtet werden.

Timo Frett und Annika Sander, Köln, befassen sich kritisch mit den von Industrieländern gespendeten medizinischen Geräten, zum Beispiel Sterilisatoren am Beispiel von Äthiopien. Fehlende Ersatzteile und mangelnde Kenntnisse des Bedienungspersonals führen entweder zu einer längerfristigen Abhängigkeit von den Geberländern - oder die Geräte rosten vor sich hin. Eine selbstkritische Beschäftigung mit diesem Thema stünde uns allen gut an. 